Case Report

\title{
Mucormycosis of the Maxillary Antrum-Diagnostic Dilemma in Chronic Sinusitis
}

\author{
Susmita Saxena and Jitin Kharbanda \\ Department of Oral Pathology and Microbiology, ESIC Dental College and Hospital, Rohini, Delhi
}

\section{Article History}

Received 15 March 2018

Accepted 23 May 2018

Keywords :

fungal, maxillary antrum,

mucormycosis

\section{Introduction}

Mucormycosis or Zygomycosis is an opportunistic, frequently fulminant fungal infection caused by the Zygomycetes class of organisms of such genera as Absidia, Mucor, Rhizomucor, and Rhizopus. Spores may be liberated into the air and inhaled by the human host. Zygomycosis may involve any areas of the body but the rhinocerebral involvement is the most relevant for dental surgeons. This fungus invades arteries and blood vessels, cause thrombi leading to obstruction of blood flow causing necrosis of hard and soft tissues. (1) Usually Mucormycosis presents as an acute infection and spreads to rhinocerebral, pulmonary, abdominal regions or maybe disseminated in form.

\section{Case Report}

A 42-year-old male patient reported with a complaint of facial swelling of the left side of 1-year duration. He had visited general practitioner repeatedly and was treated symptomatically for maxillary sinusitis and stuffy nose. Patient was a known diabetic and at the time of reporting had uncontrolled diabetes. On examination, an extraoral sinus was evident on the left zygomatic prominence of the face (Fig. 1). CT scan showed osteolytic lesion of the left maxilla (Fig. 2). Intraoral examination revealed poor oral hygiene with mobility of most of the standing teeth

Correspondence to :

Dr. Susmita Saxena

E-mail : saxroy@yahoo.com especially the left maxillary molars. Surgical intervention of the left maxillary sinus revealed excessive bone loss with exuberant blackish friable tissue on curettage which was sent for microscopic examination. Differential diagnosis of bacterial, viral or fungal osteomyelitis and malignancy of the antrum was made. Post operatively, a window was created in the oral cavity following partial maxillectomy (Fig. 3).

\section{Microscopic findings}

Ciliated pseudostratified columnar epithelial lining of the maxillary sinus with intense polymorphonuclear and mononuclear inflammatory cell infiltration with congested blood capillaries and glandular tissue was seen. Entangled mass of fungal hyphae which were flat, broad and aseptate with branching at $90^{\circ}$ to obtuse angles was noted (Fig. 4 $\& 5)$. Numerous fungal spores and sporulating hyphae were also observed. Morphologic characteristics of the hyphae suggested the diagnosis of Mucormycosis of the maxillary antrum.

The patient was advised thorough medical examination and biochemical work up which revealed high blood sugar levels.

\section{Discussion}

Zygomycosis or Mucormycosis is an opportunistic, frequently fulminant fungal infection caused by the Zygomycetes class of organisms of such genera as Absidia, Mucor, Rhizomucor, and Rhizopus. Spores may be liberated 


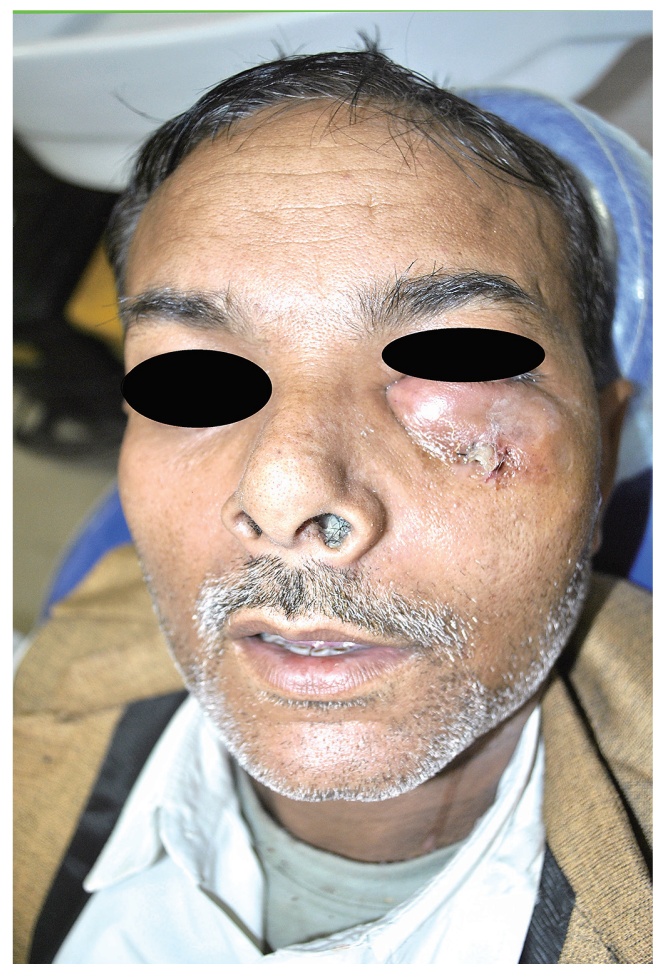

Fig. 1. Frontal view of the patient with extraoral sinus in left infraorbital region with associated swelling.

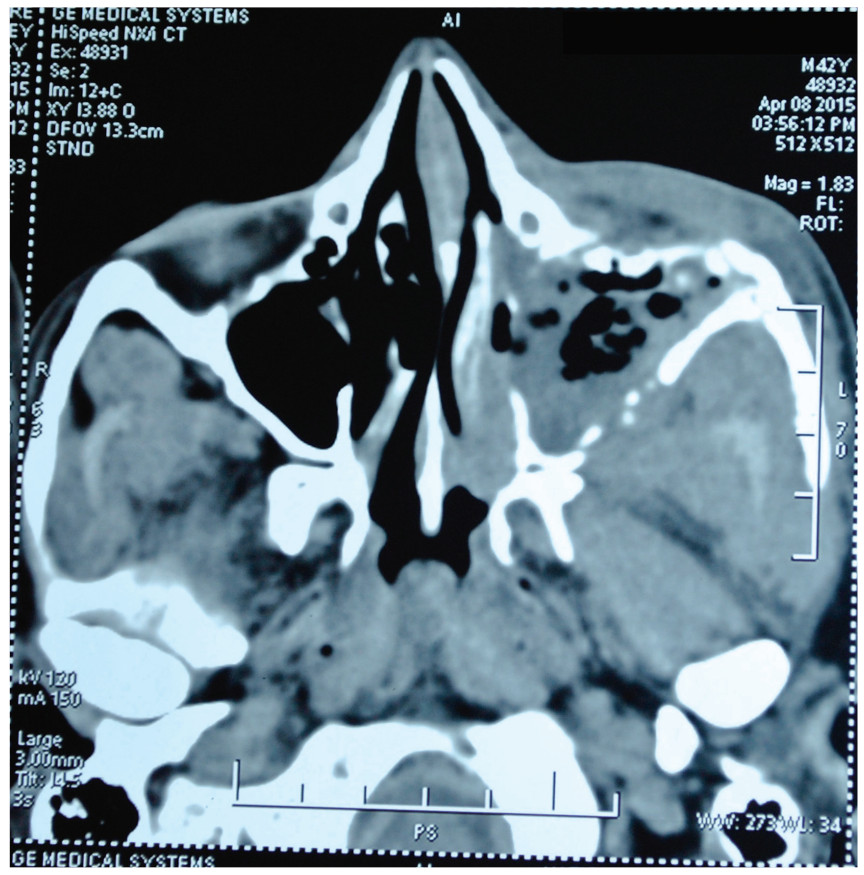

Fig. 2. CT scan shows Focal loss of bony margins of inner and outer cortex of left upper alveolus and multiple fractures involving anterior, medial and postero-lateral wall of left maxillary sinus with opacification.

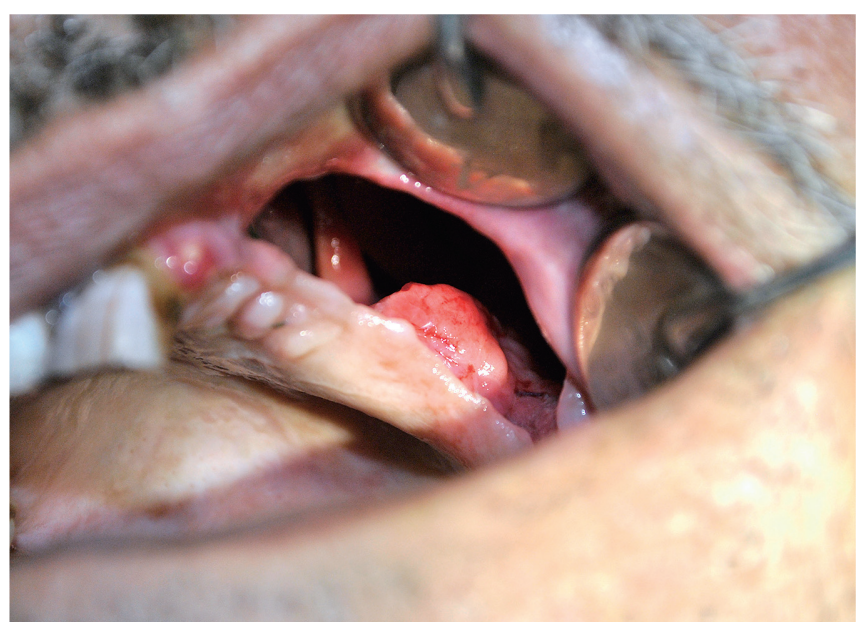

Fig. 3. Intraoral view of the left alveolar bony defect.
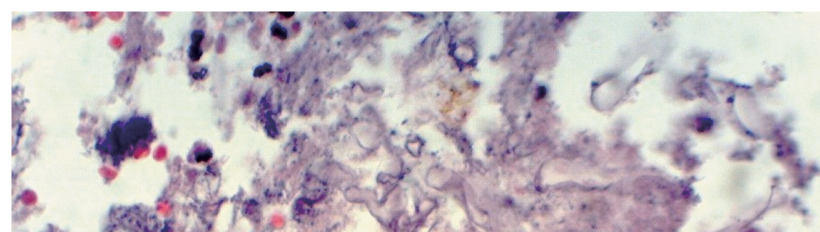

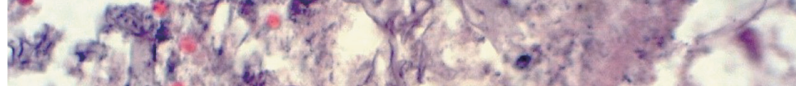

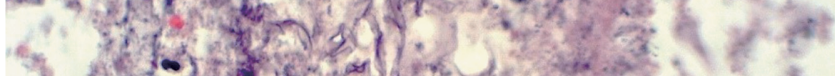

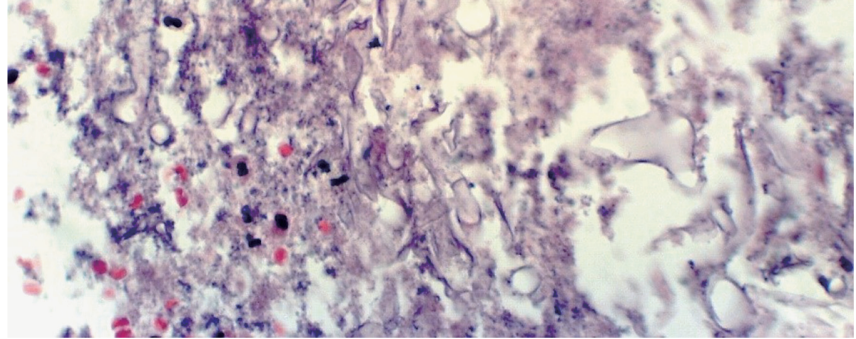

Fig. 4. H\&E stained section shows fungal hyphae and sporangia $\mathrm{x} 40 \mathrm{x}$

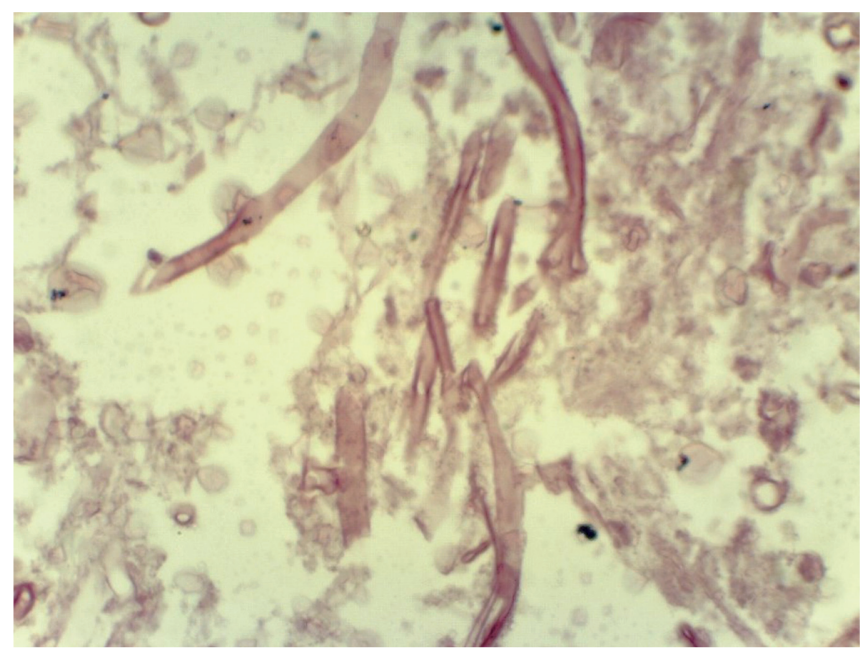

Fig. 5. Section stained with PAS stain shows flat, broad and aseptate hyphae $\mathrm{x} 40 \mathrm{x}$ magnification. 
into the air and inhaled by the human host. Differences in the epidemiology of mucormycosis seem to exist between developed and developing countries. In developed countries, the disease remains uncommon and, at present, is mostly seen in patients with diabetes mellitus and malignancies of blood undergoing chemotherapy and in transplant patients. Whereas in developing countries, especially in India, mucormycosis occurs sporadically, predominantly in patients with uncontrolled diabetes or trauma. (2) Zygomycosis may involve any areas of the body but the rhinocerebral involvement is the most relevant for dental surgeons. It is especially noted in insulin-dependent diabetics having uncontrolled diabetes and ketoacidosis. In the diabetic ketoacidotic patient, there is a high incidence of mucormycosis caused by Rhizopus oryzae, also known as Rhizopus arrhizus, because they produce the enzyme ketoreductase, which allows them to utilize the patient's ketone bodies. It is also likely that the hyperglycemia stimulates fungal growth, and the diabetic reduction in chemotaxis and phagocytic efficiency permit these otherwise innocuous organisms to proliferate. (3) This infection may affect immunocompromised patients as well and rarely affect apparently healthy individuals. If the maxillary sinus is involved, the initial presentation may be seen as intraoral swelling of the maxillary alveolar process and the palate. Massive tissue destruction may result if the condition is not treated. Radiographically the patchy effacement of the bony walls of the sinuses may create diagnostic difficulty from that of a malignancy affecting the sinus area. The condition may be misdiagnosed as maxillary sinusitis or osteomyelitis or cancrum oris delaying institution of appropriate treatment. Suspicion of mucormycosis requires a CT scan of the maxilla, orbits and brain. Evidence of intracranial brain abscesses and orbital extensions is critical and should be looked for meticulously. (4) Sinus and orbital extensions are recognized by membrane or periosteal thickenings as well as bony disruption. Histopathology of the lesional tissue shows extensive necrosis with numerous large (6 to $30 \mu \mathrm{m}$ in diameter), branching, nonseptate hyphae at the periphery. The hyphae tend to branch at 90-degree or obtuse angles and the extensive tissue destruction is caused due to the invasion of small blood vessels by the fungi. Round or ovoid sporangia are also frequently seen in the tissue section. The organisms can be cultured. Differentiation from organisms of Aspergillosis caused by genus Aspergillus shows branching septate hyphae with a tendency to branch at acute angle. Diagnosis of zygomycosis is usually based on the histopathologic findings and timely appropriate therapy must be instituted to control this fulminant infection.

Management-Patients with mucormycosis should be treated in a tertiary care center with subspecialty units experienced in the management of this condition and its underlying risk factors. Correction of the underlying abnormality, prompt initiation of liposomal amphotericin B therapy, and surgical resection are critical. Amphotericin B has proven efficacy in the treatment of mucormycosis. (5)

Control of the patient's underlying condition (like diabetic ketoacidosis) should be attempted. The prognosis however remains poor in such patients with massive tissue destruction. (6)

\section{Conclusion}

Mucormycosis is an opportunistic fungal infection which is caused by Zygomycetes class of organisms like Absidia, Mucor, Rhizomucor and Rhizopus. The rhinocerebral form is of special interest to the Dental practitioners though it may be misdiagnosed as chronic sinusitis and treated symptomatically. This highly destructive fungal infection normally affects immunocompromised patients or patients having uncontrolled diabetes with ketoacidosis. Early diagnosis can prevent massive tissue destructions and fulminant condition of the patient. Histological identification of the fungal hyphae is diagnostic of the disease and culture can be more confirmatory. Treating underlying medical condition along with debridement and institution of antifungal therapy is the advisable line of treatment.

\section{References}

1. Auluck A. Maxillary necrosis by Mucormycosis. A case report and literature review. Med Oral Patol Oral Cir Buccal, 12: E360-364, 2007.

2. Petrikkos G et al. Epidemiology and Clinical Manifestations of Mucormycosis Clinical Infectious Diseases, Volume 54, Issue suppl_1, 1 February 2012, Pages S23-S34, https://doi.org/10. 1093/cid/cir866

3. Aggarwal P, Saxena S, Bansal V. Mucormycosis of maxillary sinus. J Oral Maxillofac Pathol, 11: 66-69, 2007.

4. Marx RE, Stern D. Oral and Maxillofacial pathology: A rationale for diagnosis and treatment. 1 st ed. Quintessence Publishing Co, Inc: p.104-106, 2006.

5. McDonald PJ et al. Mucormycosis (Zygomycosis) Treatment and management. Medscape http://emedicine. medscape. com/article/222551-treatment" 
6. Neville, Damm, Alle, Bouquot. Oral and maxillofacial pathology, 2 nd ed. Saunders Company Ltd: Philadelphia, Pennsylvania; 2005. 